\title{
Double outlet right ventricle with unprotected pulmonary vasculature presenting in a woman of 65
}

\author{
Catherine Dickinson, Susan Walker, Peter Wilmshurst
}

\begin{abstract}
A 65 year old woman was admitted with confusion and dyspnoea. She had stopped attending the hospital 20 years earlier because she felt that doctors could not help her. The notes showed that during the preceding 45 years she had been investigated at several cardiac centres for cyanosis and clubbing. Cardiac catheterisation had been performed twice but the diagnosis was not clear. She was believed to have a septal defect (atrial or ventricular or both), but the defect was regarded as inoperable because of pulmonary hypertension. She was married and had had three pregnancies: two miscarried but one went to term and a healthy child was delivered. She was on no medication.
\end{abstract}

On examination she was cyanosed and showed clubbing. Rhythm was fast atrial fibrillation. Right ventricular and pulmonary artery impulses were prominent. There was a loud systolic murmur at the left sternal edge and signs of right and left heart failure. The electrocardiogram showed right bundle branch block. Chest $x$ ray showed cardiomegaly with pulmonary plethora (figure).

With digoxin, amiodarone, diuretics, and oxygen treatment her confusion and signs of heart failure resolved, her atrial fibrillation was controlled, and the degree of desaturation and dyspnoea improved.

Echocardiography and cardiac catheterisation were performed. These showed normal atrial situs with no interatrial communication but a left superior vena cava drained to the right atrium via the coronary sinus. There was a double outlet right ventricle with a large ventricular septal defect and only a small left ventricle. Simultaneous pressures in the peripheral pulmonary arteries and ascending aorta were $110 / 25$ and $125 / 60 \mathrm{~mm} \mathrm{Hg}$ respectively. There was no stenosis of the pulmonary artery branch and repeated pullbacks across the pulmonary valve showed gradients of $0-10$ $\mathrm{mm} \mathrm{Hg}$. Saturations in the pulmonary veins, which were reached by crossing the ventricular septal defect and passing retrogradely across the mitral valve, were $96 \%$.

She was discharged well, if mildly dyspnoeic and significantly cyanosed on $20 \mathrm{mg}$ frusemide, $2.5 \mathrm{mg}$ amiloride, $0.25 \mathrm{mg}$ digoxin, and $200 \mathrm{mg}$ amiodarone daily.

As far as we are aware, this patient is unique. She has lived a nearly normal life, including child-bearing, and, despite having a condition that is generally believed to have a universally poor prognosis has survived to 65 and is taking relatively little medication.

Death in infancy from congestive heart failure or prematurely from the development of pulmonary vascular occlusive disease is the rule in patients with double outlet right ventricle with pulmonary vasculature unprotected by pulmonary valve or pulmonary artery branch stenosis. Though she has a systemic level of pulmonary systolic pressure her pulmonary artery diastolic pressure is only slightly increased. The considerable pulmonary artery pulse pressure without evidence of significant pulmonary valve regurgitation seems to indicate that her pulmonary vascular resistance has risen less than is usual in similar cases. Despite 65 years of exposure to high pulmonary artery pressures she has been relatively resistant to pulmonary vascular occlusive disease.

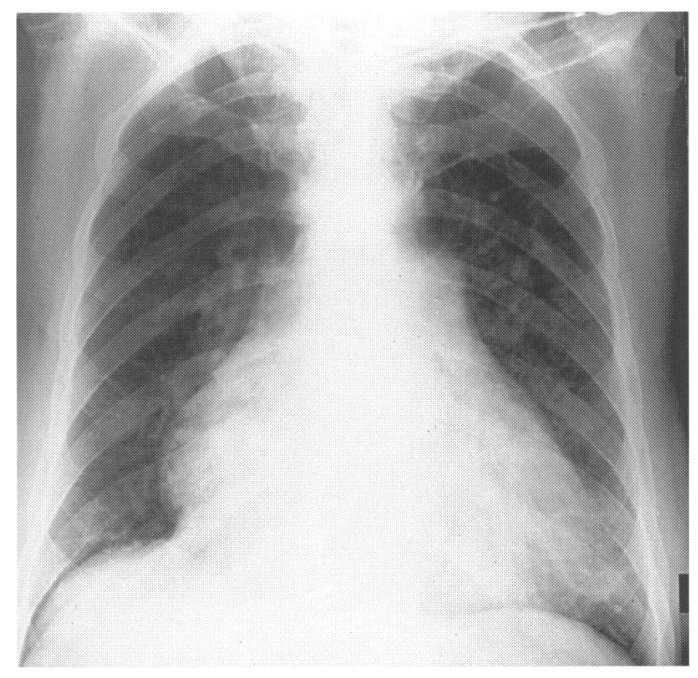

Chest $x$ ray showing cardiomegaly, prominent main pulmonary arteries, and pulmonary plethora 